\title{
Neuroleptic Malignant Syndrome
}

National Institute of Neurological Disorders and Stroke (NINDS)

\section{Source}

National Institute of Neurological Disorders and Stroke (NINDS). Neuroleptic Malignant

Syndrome Information Page.

Neuroleptic malignant syndrome is a life-threatening, neurological disorder most often caused by an adverse reaction to neuroleptic or antipsychotic drugs. Symptoms include high fever, sweating, unstable blood pressure, stupor, muscular rigidity, and autonomic dysfunction. In most cases, the disorder develops within the first 2 weeks of treatment with the drug; however, the disorder may develop any time during the therapy period. The syndrome can also occur in people taking anti-Parkinsonism drugs known as dopaminergics if those drugs are discontinued abruptly. 